PROCEEDINGS OF THE

AMERICAN MATHEMATICAL SOCIETY

Volume 125, Number 2, February 1997, Pages 555-567

S 0002-9939(97)03507-7

\title{
SMOOTH REPRESENTATION OF A PARAMETRIC POLYHEDRAL CONVEX SET WITH APPLICATION TO SENSITIVITY IN OPTIMIZATION
}

\author{
DINH THE LUC
}

(Communicated by Joseph S. B. Mitchell)

\begin{abstract}
We show in this paper that if a polyhedral convex set is defined by a parametric linear system with smooth entries, then it possesses local smooth representation almost everywhere. This result is then applied to study the differentiability of the solutions and the marginal functions of several classes of parametric optimization problems.
\end{abstract}

\section{INTRODUCTION}

Let $M$ be a polyhedral convex set in a finite dimensional space $X$, i.e. $M$ can be expressed as the set of solutions to some finite system of linear inequalities of the form

$$
a_{i}(x)+\alpha_{i} \leq 0, \quad i=1, \ldots, k,
$$

where $a, \ldots, a_{k}$ are vectors of the dual space $X^{\prime} ; \alpha, \ldots, \alpha_{k}$ are real numbers. Sometimes we shall write $\left\langle a_{i}, x\right\rangle$ instead of $a_{i}(x)$. It is known that $M$ is a finitely generated convex set [14] which means that there exist vectors $v, \ldots, v_{m} \in X$ and an integer $l, 0<l \leq m$, such that

$$
M=\left\{x \in X: x=\sum_{i}^{m} \lambda_{i} v_{i}, \sum_{i}^{l} \lambda_{i}=1, \lambda_{i} \geq 0, i=1, \ldots, m\right\} .
$$

In other words, $M$ can be represented by the points $v, \ldots, v_{l}$ and the directions $v_{l}, \ldots, v_{m}$. Now assume that $a, \ldots, a_{k}$ and $\alpha, \ldots, \alpha_{k}$ depend on a parameter $\omega$ from a parameter space $\Omega$ which is supposed to be an open subset of a finite dimensional space. Then $M, m, l, v, \ldots, v_{m}$ are all functions of $\omega$. It is interesting to know whether one can choose smooth representing points and directions if the entries $a, \ldots, a_{k}$ and $\alpha, \ldots, \alpha_{k}$ are smooth. This question is important for the following reason. Let $F$ be a set-valued map from $\Omega$ to $X$. A point-valued map $f$ from $\Omega$ to $X$ is said to be a selection of $F$ if $f(\omega) \in F(\omega)$ for every $\omega \in \Omega$. Michael's theorem, Castaing-Valadier's theorem, Cellina's theorem, Lojasiewicz's

Received by the editors January 25, 1995 and, in revised form, May 17, 1995.

1991 Mathematics Subject Classification. Primary 52A20, 90C31.

Key words and phrases. Polyhedral convex set, representing point and direction, sensitivity analysis, parametric linear problem, parametric concave problem, parametric polyhedral problem.

The author is on leave from the Institute of Mathematics, Hanoi, Vietnam. 
theorem (see [1]) are the best-known results on the existence of continuous, measurable, Lipschitz selections. Moreover, under a suitable hypothesis one can find a continuous, measurable, Lipschitz parametrization, i.e. a single-valued function $f$ from $\Omega \times \Lambda$ to $X$, where $\Lambda$ is a control space, such that $F(\omega)=\bigcup\{f(\omega, \lambda): \lambda \in \Lambda\}$. Now, what about differentiable selection and parametrization? When does $F$ admit a differentiable selection and a differentiable parametrization? The answers to these questions are crucial for sensitivity analysis in many problems of applied analysis. Of particular interest is the case when $F$ represents a solution set to generalized equations. Until now many results have been obtained for the situations when $F$ is locally single valued (see [5], [13] and the references given therein), and quite recently significant efforts have been made to treat the case where $F$ is set valued (see [7], [9]). The paper [7] tackles the problem in a very general setting and concentrates the study on the proto-differentiability of the set valued map $F$.

The purpose of the present paper is to prove the existence of local smooth representing vectors for $M$ introduced above, thereby showing that $M$ admits a local smooth parametrization as well as a local smooth selection. The result is then applied to investigate the differentiability of the solutions and the marginal functions of several parametric optimization problems.

The paper is structured as follows. In the next section we prove that one can find an open set $U \subseteq U, m$ functions $v, \ldots, v_{m} \in C^{r}(U, X)$, and an integer $l$, $0<l \leq m$, where $l$ and $m$ do not depend on $\omega$, such that

$$
M(\omega)=\left\{x \in X: x=\sum_{i}^{m} \lambda_{i} v_{i}(\omega), \sum_{i}^{l} \lambda_{i}=1, \lambda_{i} \geq 0, i=1, \ldots, m\right\},
$$

for every $\omega \in U$. In Section 3, this result is applied to study a parametric linear optimization problem and its dual. As a consequence, a parametric version of Farkas' theorem is obtained. Section 4 is devoted to parametric concave and quasiconcave problems with linear constraints. In the last section, a parametric polyhedral convex problem is investigated.

\section{A SMOoth REPRESEntation Result}

Throughout this paper, $a, \ldots, a_{p} q$ are supposed to be in $C^{r}(\Omega, X)$ and $\alpha, \ldots$, $\alpha_{p} q_{q}$ are supposed to be in $C^{r}(\Omega, R), r \geq 0$. Let $J:=\left\{j, \ldots, j_{l}\right\} \subseteq\{1, \ldots, p+$ $q\}$. Then $A_{J}(\omega)$ denotes the matrix whose rows are $a_{j_{1}}(\omega), \ldots, a_{j_{l}}(\omega)$, and $\alpha_{J}(\omega)$ denotes the vector whose components are $\alpha_{j_{1}}(\omega), \ldots, \alpha_{j_{l}}(\omega)$. The cardinality of $J$ is denoted by $|J|$ (here $|J|=l$ ). The polyhedral convex set $M(\omega)$ is defined by the following system of inequalities and equations:

$$
\begin{aligned}
& \left\langle a_{i}(\omega), x\right\rangle+\alpha_{i}(\omega) \leq 0, \quad i=1, \ldots, p ; \\
& \left\langle a_{j}(\omega), x\right\rangle+\alpha_{j}(\omega)=0, \quad j=p+1, \ldots, p+q .
\end{aligned}
$$

Recall that a set-valued map $F$ from $\Omega$ to $X$ is said to be lower (respectively upper) semicontinuous at $\omega \in \Omega$ if for every $x \in F(\omega)$, for every neighborhood $V$ of $x$ (resp., for every open set $V$ containing $F(\omega)$ ) in $X$, there exists a neighborhood $U$ of $\omega$ in $\Omega$ such that $F(\omega) \cap V \neq \varnothing$ (resp., $F(\omega) \subseteq V$ ) whenever $\omega \in U$. It is said to be closed at $\omega \in \Omega$ if $x \in F(\omega)$ whenever $x=\lim _{n \rightarrow \infty} x_{n}$, where $x_{n} \in F\left(\omega_{n}\right)$, $\omega_{n} \in \Omega, n=1,2, \ldots$, and $\lim _{n \rightarrow \infty} \omega_{n}=\omega$. If $F$ is lower semicontinuous and closed at every point of $U$, we say that it is continuous on $U$ (see [1], [3], [11]). In the literature $F$ is called continuous if it is simultaneously upper and lower 
semicontinuous. The two definitions are equivalent provided $F$ has compact values. For the case of noncompact values, upper semicontinuity is a very restrictive notion, so we are not going to deal with it in our study. In the sequel to avoid considering separately the case of empty values, we shall also call $F$ continuous on $U$ if $F(\omega)=\varnothing$ for every $\omega \in U$.

The map $\omega \mapsto M(\omega)$ is always closed, but not lower semicontinuous in general (and never upper semicontinuous if $M(\omega)$ is unbounded and distinct from a constant map). There exists an extensive literature on lower semicontinuity criteria for this kind of maps (see e.g. [2], [4], [12]). However, in this section, we shall need the following strong result recently established in [9].

Lemma 2.1 ([9]). For every open set $U \subseteq \Omega$, there exists an open subset $U \subseteq U$ such that $M$ is continuous on $U$.

Let $A_{J_{1}}^{-}$denote the inverse of the matrix $A_{J_{1}}$. We shall not indicate the transposition of a vector in the multiplication with a matrix, hoping that no confusion occurs by understanding a vector as a row or as a column.

Lemma 2.2. Assume that $M$ is continuous on $U$ and $M(\omega)$ possesses at least one vertex for some $\omega \in U$. Then there exists an open subset $U \subseteq U$, and $k$ subsets $J, \ldots, J_{k} \subseteq\{1, \ldots, p+q\}$ with $|J|=\cdots=\left|J_{k}\right|=\operatorname{dim} X$ such that for every $\omega \in U$, the polyhedral convex set $M(\omega)$ possesses exactly $k$ vertices $v(\omega), \ldots, v_{k}(\omega)$ defined by equations:

$$
v_{i}(\omega)=A_{J_{i}}^{-}(\omega)\left(-\alpha_{J_{i}}(\omega)\right), \quad i=1, \ldots, k .
$$

Proof. Let us denote by $d(\omega)$ the number of vertices of $M(\omega)$. We show that for each $\omega \in U$ there is a neighborhood $U \subseteq U$ of $\omega$ such that $d(\omega) \geq d(\omega)$ for all $\omega \in U$. In fact, since $d(\omega)$ is finite, there can be found a positive $t$ such that all the vertices of $M(\omega)$ are located in the interior of the box $B:=\{x=$ $\left.\left(x, \ldots, x^{n}\right) \in R^{n}: \max _{i}, \ldots, n\left|x^{i}\right| \leq t\right\}$. We claim that around each vertex of $M(\omega)$ must at least one vertex of $M(\omega)$ be found, if $\omega$ is sufficiently close to $\omega$, thereby establishing the required inequality. Indeed, let $v$ be a vertex of $M(\omega)$. By the lower semicontinuity of $M$ there is $x_{\omega} \in M(\omega)$ such that $\lim _{\omega \rightarrow \omega_{0}} x_{\omega}=v$. One can express $x_{\omega}=y_{\omega}+z_{\omega}$ where $y_{\omega}$ is a convex combination of vertices of $M(\omega)$ and $z_{\omega}$ is a direction of $M(\omega)$. It is evident that $\lim _{\omega \rightarrow \omega_{0}} z_{\omega}=0$. Otherwise, one should choose a sequence $\left\{y_{\omega_{i}}\right\}_{i}^{\infty} \subseteq B$ converging to some $y \in M(\omega)$, a sequence $\left\{z_{\omega_{i}}\right\}_{i}^{\infty}$ converging to some nonzero direction $z$ of $M(\omega)$, as $\omega_{i}$ tends to $\omega$ and arrive at a contradiction $v=y+z$ (remembering that $v$ is a vertex). Moreover, there is at least one vertex $v(\omega)$ in the convex combination $y_{\omega}$ such that $\lim _{\omega \rightarrow \omega_{0}} v(\omega)=v$. Otherwise, since the number of vertices of $M(\omega)$ is majorized by $C_{p q}^{n}$, there should exist a sequence $\left\{y_{\omega_{i}}\right\}_{i}^{\infty}$ converging to a convex combination of some points of $M(\omega)$ which are distinct from $v$ and we again should arrive at a contradiction that the vertex $v$ is represented by a convex combination of other points of $M(\omega)$.

Let $\omega$ be a point which maximizes $d(\omega)$ on $U$. Such a point exists because $d(\omega)$ is majorized, as mentioned above. Then there is a neighborhood $U \subseteq U$ of $\omega$ such that $d(\omega)=d(\omega)$ for all $\omega \in U$. Let $v_{i}(\omega), i=1, \ldots, d(\omega)$ be the vertices of $M(\omega)$. We may assume that $U$ is so small that in a small neighborhood of each vertex $v_{i}(\omega)$ there is exactly one vertex, say $v_{i}(\omega)$ of $M(\omega)$. For every fixed index $i$, let

$$
J\left(v_{i}(\omega)\right)=\left\{j \in\{1, \ldots, p+q\}:\left\langle a_{j}(\omega), v_{i}(\omega)\right\rangle+\alpha_{j}(\omega)=0\right\} .
$$


Then $\left|J\left(v_{i}(\omega)\right)\right| \geq n$ and evidently, if an index $j$ does not belong to $J\left(v_{i}(\omega)\right)$, neither does it belong to $J\left(v_{i}\left(\omega^{\prime}\right)\right)$ when $\omega^{\prime}$ is sufficiently close to $\omega$. In other words $J\left(v_{i}\left(\omega^{\prime}\right)\right) \subseteq J\left(v_{i}(\omega)\right)$ for every $\omega^{\prime}$ in a sufficiently small neighborhood of $\omega$. Choose a point $\omega$ which minimizes the number $\left|J\left(v_{i}(\omega)\right)\right|$ on $U$. Then one can find a neighborhood $U \subseteq U$ of $\omega$ such that $J\left(v_{i}(\omega)\right)=J\left(v_{i}(\omega)\right)$ for all $\omega \in U$. Pick any $n$ indices from the set $J\left(v_{i}(\omega)\right)$ with the property that the corresponding vectors $a_{j}(\omega)$ form a linearly independent system. There is a smaller neighborhood $U \subseteq U$ where these vectors are still linearly independent. The vertex $v_{i}(\omega)$, $\omega \in U$, is then determined by the equation given in the lemma.

If $M(\omega) \neq \varnothing$ has no vertices, its lineality space denoted by $L(\omega)$ is nontrivial.

Lemma 2.3. Assume that $M$ is nonvoid-valued and continuous on $U$. Then there exists an open subset $U \subseteq U$ and a subset $J \subseteq\{1, \ldots, p+q\}$ such that for every $\omega \in U, L(\omega)$ is the orthogonal space to the space spanned by the vectors $\left\{a_{i}(\omega): i \in J\right\}$. Consequently, if $s:=\operatorname{dim} X-|J|>0$, there can be found $s$ functions $u, \ldots, u_{s} \in C^{r}(U, X)$ such that $u(\omega), \ldots, u_{s}(\omega)$ form a basis of $L(\omega)$, for every $\omega \in U$.

Proof. Let $\omega \in U$. We show first that there is a neighborhood $U$ of $\omega$ in $U$ such that $\operatorname{dim} L(\omega) \leq \operatorname{dim} L(\omega)$, for all $\omega \in U$. In fact, since the lineality space $L(\omega)$ is defined by equations

$$
\left\langle a_{i}(\omega), x\right\rangle=0, \quad i=1, \ldots, p+q,
$$

one has

$$
\operatorname{dim} L(\omega)=\operatorname{dim} X-\operatorname{rank}\left\{a(\omega), \ldots, a_{p} \quad q(\omega)\right\} .
$$

For a fixed $\omega \in U$, one can find a neighborhood $U$ of $\omega$ in $U$ such that

$$
\operatorname{rank}\left\{a(\omega), \ldots, \alpha_{p} q(\omega)\right\} \leq \operatorname{rank}\left\{a(\omega), \ldots, a_{p} q(\omega)\right\},
$$

whenever $\omega \in U$. Hence $\operatorname{dim} L(\omega) \leq \operatorname{dim} L(\omega), \omega \in U$. By this, if we take $\omega \in U$ with the property that

$$
\operatorname{dim} L(\omega)=\min \{\operatorname{dim} L(\omega): \omega \in U\},
$$

then $\operatorname{dim} L(\omega)=\operatorname{dim} L(\omega)$ for every $\omega \in U$. Let $J \subseteq\{1, \ldots, p+q\}$ be an index subset such that $\left\{a_{i}(\omega): i \in J\right\}$ is a maximal linearly independent subsystem of the system $\left\{a_{i}(\omega): i=1, \ldots, p+q\right\}$. It is clear that

$$
L(\omega)=\left\{x \in X:\left\langle a_{i}(\omega), x\right\rangle=0, i \in J\right\},
$$

and that $\operatorname{dim} L(\omega)=\operatorname{dim} X-|J|$. We have $s=\operatorname{dim} L(\omega)$. By taking a smaller neighborhood if necessary, we may assume that $\left\{a_{i}(\omega): i \in J\right\}$ is still a linearly independent system whenever $\omega \in U$. Since $\operatorname{dim} L(\omega)=\operatorname{dim} L(\omega)$, one has

$$
L(\omega)=\left\{x \in X:\left\langle a_{i}(\omega), x\right\rangle=0, i \in J\right\},
$$

and hence $L(\omega)$ is the orthogonal space to the space spanned by $\left\{a_{i}(\omega): i \in J\right\}$.

Now assume $s>0$. Let $A(\omega)$ be a nonsingular $|J| \times|J|$-submatrix of $A_{J_{0}}(\omega)$. Without loss of generality, one may also assume that the matrix $A(\omega)$ is nonsingular for $\omega \in U$. To facilitate the writing, assume that $A_{J_{0}}(\omega)=(A(\omega) \mid B(\omega))$. Denote by $e_{i} \in R^{s}, i=1, \ldots, s$, the vectors whose unique nonzero component is equal to 1 and is at the $i$-th place. Then it is evident that the vectors

$$
u_{i}(\omega)=\left((A(\omega))^{-}\left(-B(\omega) e_{i}\right), e_{i}\right), \quad i=1, \ldots, s,
$$


form a basis of $L(\omega)$. Trivially, the functions $u, \ldots, u_{s}$ belong to $C^{r}(U, X)$. The proof is complete.

Now we are able to give the main result of the paper.

Proposition 2.1. Assume as before that $a, \ldots, a_{p} q \in C^{r}\left(\Omega, X^{\prime}\right)$ and $\alpha, \ldots$, $\alpha_{p} q_{q} \in C^{r}(\Omega, R)$. Then for every open set $U \subseteq \Omega$, there exists an open subset $U \subseteq U$ such that either $M$ is empty-valued on $U$, or one can find $m$ functions $v, \ldots, v_{m} \in C^{r}(U, X)$ and an integer $k, 0<k \leq m$, such that

$$
M(\omega)=\left\{x \in X: x=\sum_{i}^{m} \lambda_{i} v_{i}(\omega), \sum_{i}^{k} \lambda_{i}=1, \lambda_{i} \geq 0, i=1, \ldots, m\right\},
$$

for every $\omega \in U$.

Proof. By Lemma 2.1, we may assume that $M$ is continuous on $U$. If $M(\omega)=\varnothing$ for all $\omega \in U$, we are done. If not, by the continuity there is a neighborhood in $U$ where the values of $M$ are nonempty. Therefore without loss of generality, it can be assumed that $M(\omega) \neq \varnothing$ for all $\omega \in U$. Let $\omega \in U$ be a point where $\operatorname{dim} L(\omega)$ is minimal among $\operatorname{dim} L(\omega), \omega \in U$.

If $\operatorname{dim} L(\omega)=0$, then $M(\omega)$ possesses at least one vertex. Put $M(\omega):=M(\omega)$ and $s:=0$. If $\operatorname{dim} L(\omega)>0$, by virtue of Lemma 2.3, there is a neighborhood $U$ of $\omega$ in $U$ and $s$ functions $u, \ldots, u_{s} \in C^{r}(U, X)$ such that $L(\omega)$ is generated by the vectors $u(\omega), \ldots, u_{s}(\omega)$ for each $\omega \in U$. Let

$$
M(\omega):=M(\omega) \cap\{x \in X:\langle u(\omega), x\rangle=0, i=1, \ldots, s\} .
$$

In other words, $M(\omega):=M(\omega) \cap L^{\perp}(\omega)$, where $L^{\perp}(\omega)$ is the orthogonal space to $L(\omega)$. It is known (see [14]) that $M(\omega)=M(\omega)+L(\omega)$, and $M(\omega)$ has no lines which means that it has at least one vertex (extreme point). Using Lemma 2.1 we may assume that the map $M$ is continuous on $U$. By Lemma 2.2, one can find an open subset $U \subseteq U$ and $k$ functions $v, \ldots, v_{k} \in C^{r}(U, X)$ such that for every $\omega \in U$, the polyhedral set $M(\omega)$ has exactly $k$ vertices: $v(\omega), \ldots, v_{k}(\omega)$. Recall that $v$ is a recession direction (or say simply a direction) of $M(\omega)$ if $M(\omega)+t v \subseteq$ $M(\omega)$ for every $t \geq 0$ (see [14]), i.e. it is a solution to the system

$$
\begin{aligned}
\left\langle a_{i}(\omega), v\right\rangle & \leq 0, & i & =1, \ldots, p ; \\
\left\langle a_{j}(\omega), v\right\rangle & =0, \quad & j & =p+1, \ldots, p+q .
\end{aligned}
$$

If for some $\bar{\omega} \in U$, the set $M(\bar{\omega})$ has no nonzero recession directions, i.e. it is a polytope, then by the continuity of $M$ there is a neighborhood $\bar{U}$ of $\bar{\omega}$ in $U$ such that $M(\omega)$ is a polytope, too, $\omega \in \bar{U}$. In this event, $v, \ldots, v_{k}, u, \ldots, u_{s},-u, \ldots,-u_{s}$ are the functions we need to represent $M$. In the other case, we fix any $\bar{\omega}$ in $U$ and denote the recession cone of $M(\omega)$ by $\operatorname{Rec} M(\omega)$. We claim that one can find a neighborhood $\bar{U}$ of $\bar{\omega}$ in $U$ and a vector $d \in X^{\prime}$ such that $\langle d, v\rangle>0$ for every $v \in \operatorname{Rec} M(\omega) \backslash\{0\}, \omega \in \bar{U}$. Indeed, since $\operatorname{Rec} M(\omega)$ is convex, closed and contains no lines, there is a vector $d \in X^{\prime}$ such that the latter inequality holds for every $v \in \operatorname{Rec} M(\omega) \backslash\{0\}$. If the statement was not true, one should find a sequence $\left\{\omega_{i}\right\}_{i}^{\infty}$ converging to $\omega$ and $v_{i} \in \operatorname{Rec} M\left(\omega_{i}\right)$ such that $\left\langle d, v_{i}\right\rangle \leq 0$ for all $i \geq 1$. It may be assumed that $\left\{v_{i}\right\}_{i}^{\infty}$ converges to some nonzero vector $v$ which is a direction of $M(\omega)$ due to the continuity of $M$ and one should have a contradiction $\langle d, v\rangle \leq 0$. 
Let us consider the polytopes

$$
M(\omega):=\operatorname{Rec} M(\omega) \cap\{x \in X:\langle d, x\rangle=1\},
$$

for $\omega \in \bar{U}$. In view of Lemma 2.2, we can find an open subset $U \subseteq \bar{U}$ and $l$ functions $v_{k}, \ldots, v_{k} l \in C^{r}(U, X)$ such that for every $\omega \in U$, the polytope $M(\omega)$ has exactly $l$ vertices $v_{k}(\omega), \ldots, v_{k} l(\omega)$. It is evident that

$$
\operatorname{Rec} M(\omega)=\left\{x \in X: x=\sum_{i}^{k} \lambda_{i} v_{i}(\omega), \lambda_{i} \geq 0, i=k+1, \ldots, k+l\right\},
$$

and also

$$
M(\omega)=\left\{x \in X: x=\sum_{i}^{k} \lambda_{i} v_{i}(\omega), \sum_{i}^{k} \lambda_{i}=1, \lambda_{i} \geq 0, i=1, \ldots, k+l\right\} .
$$

The functions $v, \ldots, v_{k} l, u, \ldots, u_{s},-u, \ldots,-u_{s}$ are those we look for. The proof is complete.

We conclude this section by observing that under the assumption of Proposition $2.1, \lambda:=\left(\lambda, \ldots, \lambda_{m}\right)$ can be regarded as a control and a parametrization of $M$ can be given by

$$
f(\omega, \lambda):=\sum_{i}^{m} \lambda_{i} v_{i}(\omega)
$$

This function is of class $C^{r}$ on $U$ with respect to the variable $\omega$ for every fixed control. The control space $\Lambda$ consists of vectors $\lambda$ satisfying $\sum_{i}^{k} \lambda_{i}=1, \lambda_{i} \geq 0$, $i=1, \ldots, m$.

Observe also that if $M(\omega)$ possesses vertices, the lineality space $L(\omega)$ must be zero. Moreover, all the vertices of $M(\omega)$ must be among $v, \ldots, v_{k}$; while all its extreme directions must appear among $v_{k}, \ldots, v_{k} l$. Nonextreme points and directions of the system $\left\{v, \ldots, v_{k} l\right\}$ become superfluous in the representation of $M(\omega)$. Thus, in this case $M(\omega)$ may be represented by extreme points and extreme directions only.

\section{PARAMETRIC LineAR PROBlem}

Let us consider the following parametric linear optimization problem, denoted by $P(\omega)$ :

$$
\begin{aligned}
\inf & \langle c(\omega), x\rangle, \\
\text { subject to } & x \in M(\omega)
\end{aligned}
$$

where $c \in C^{r}\left(\Omega, X^{\prime}\right) ; M$ is as in the previous section. The marginal function of the problem is defined as

$$
\varphi(\omega):=\inf \{\langle c(\omega), x\rangle: x \in M(\omega)\},
$$

and the solution map is defined as

$$
S(\omega):=\{x \in M(\omega):\langle c(\omega), x\rangle=\varphi(\omega)\},
$$

whenever $\varphi(\omega)$ is finite. The following proposition is a strengthened version of a result previously given in [9]. 
Proposition 3.1. Assume that $M(\omega) \neq \varnothing$ for all $\omega$ from an open set $U \subseteq \Omega$. Then there exists an open subset $U \subseteq U$ with the following property: either one can find a function $v \in C^{r}(\Omega, X)$ such that for every $\omega \in U, v(\omega)$ is a recession direction of $M(\omega)$ and

$$
\langle c(\omega), v(\omega)\rangle<0,
$$

in which case $\varphi(\omega)=-\infty$; or one can find l functions $v, \ldots, v_{l} \in C^{r}(U, X)$ and an integer $k, 0<k \leq l$, such that

$$
\begin{gathered}
S(\omega)=\left\{x \in X: x=\sum_{i}^{l} \lambda_{i} v_{i}(\omega), \sum_{i}^{k} \lambda_{i}=1, \lambda_{i} \geq 0, i=1, \ldots, l\right\}, \\
\left\langle c(\omega), v_{j}(\omega)\right\rangle=0, \quad j=k+1, \ldots, l, \\
\left\langle c(\omega), v_{i}(\omega)\right\rangle=\varphi(\omega), \quad i=1, \ldots, k,
\end{gathered}
$$

for every $\omega \in U$. In particular, $\varphi$ is of class $C^{r}$ on $U$.

Proof. Use Proposition 2.1 to have smooth representing points and directions of $M$ on an open subset $U$ of $U$. If for some $\omega \in U$, and for some $j, k<j \leq m$, one has $\left\langle c(\omega), v_{j}(\omega)\right\rangle<0$, then in a sufficiently small neighborhood $U$ of $\omega$ in $U$, it is also true that $\left\langle c(\omega), v_{j}(\omega)\right\rangle<0, \omega \in U$. This shows that $\varphi(\omega)=-\infty$, $\omega \in U$. Now, we may assume that $\left\langle c(\omega), v_{j}(\omega)\right\rangle \geq 0$, for all $j=k+1, \ldots, m$, and for all $\omega \in U$. In this case, evidently $\varphi(\omega)$ is finite. Observe that if at some $\omega \in U$, and for some $j, k<j \leq m$, one has $\left\langle c(\omega), v_{j}(\omega)\right\rangle>0$, then as above, in a sufficiently small neighborhood $U$ of $\omega$ in $U$, it is also true that $\left\langle c(\omega), v_{j}(\omega)\right\rangle>0$, $\omega \in U$. Thus, without loss of generality, it can be assumed that $\left\langle c(\omega), v_{i}(\omega)\right\rangle=0$, for $i=k+1, \ldots, l$, and $\left\langle c(\omega), v_{j}(\omega)\right\rangle>0$, for $j=l+1, \ldots, m$, for some $l$ with $k+1 \leq l \leq m$, whenever $\omega$ in $U$.

Denote by $\bar{J}(\omega) \subseteq\{1, \ldots, k\}$ the index set of those representing points which minimize the objective function $c(\omega)$ on $M(\omega)$, i.e.

$$
\varphi(\omega)=\left\langle c(\omega), v_{i}(\omega)\right\rangle\left\langle\left\langle c(\omega), v_{j}(\omega)\right\rangle\right.
$$

for $i \in \bar{J}(\omega), j \in\{1, \ldots, k\} \backslash \bar{J}(\omega)$. It is clear that $\bar{J}\left(\omega^{\prime}\right) \subseteq \bar{J}(\omega)$ if $\omega^{\prime}$ is sufficiently close to $\omega$. Consequently, if we take $\omega$ in $U$ to be a point with

$$
|\bar{J}(\omega)|=\min \{|\bar{J}(\omega)|: \omega \in U\},
$$

then in a sufficiently small neighborhood $U$ of $\omega$ in $U$ one must have

$$
\varphi(\omega)=\left\langle c(\omega), v_{i}(\omega)\right\rangle<\left\langle c(\omega), v_{j}(\omega)\right\rangle
$$

for all $i \in \bar{J}(\omega), j \in\{1, \ldots, k\} \backslash \bar{J}(\omega), \omega \in U$.

With a suitable change of indices and of the numbers $k$ and $l$, the solution set $S(\omega)$ is then presented in the formula of the proposition.

Corollary 3.1. The set of the points where $\varphi$ is finite and not of class $C^{r}$ is nowhere dense.

Proof. It is immediate from the previous proposition.

Now let us denote by $D(\omega)$ the dual of the problem $P(\omega)$. It is also a parametric linear problem which is given in the following form:

$$
\begin{aligned}
\sup & \langle\alpha(\omega), y\rangle, \\
\text { subject to } & y \in \widehat{M}(\omega)
\end{aligned}
$$


where $\alpha(\omega):=\left(\alpha(\omega), \ldots, \alpha_{p} \quad q(\omega)\right)$ and

$$
\widehat{M}:=\left\{y:=\left(y, \ldots, y_{p} q\right) \in Y: y A(\omega)+c(\omega)=0, y_{i} \leq 0, i=1, \ldots, p\right\}
$$

(here $Y$ is the $(p+q)$-dimensional space, $A$ is the matrix whose rows are $a, \ldots$, $\left.\begin{array}{ll}a_{p} & q\end{array}\right)$. The marginal function $\psi$ and the solution map $\widehat{S}$ of the dual problem are defined in a similar way as those of the primal problem. Under the common hypothesis that $\alpha, \ldots, \alpha_{p} \quad$ and $a, \ldots, a_{p} q$ are of class $C^{r}$, we have the following result for the dual parametric problem.

Proposition 3.2. Assume that $\widehat{M}(\omega) \neq \varnothing$ for all $\omega$ from an open set $U \subseteq \Omega$. Then there exists an open subset $U \subseteq U$ with the following property: either one can find a function $u \in C^{r}(\Omega, Y)$ such that for every $\omega \in U, u(\omega)$ is a recession direction of $\widehat{M}(\omega)$ and

$$
\langle\alpha(\omega), u(\omega)\rangle>0,
$$

in which case $\psi(\omega)=\infty$; or one can find $n$ functions $u, \ldots, u_{n} \in C^{r}(U, Y)$ and an integer $m, 0<m \leq n$, such that

$$
\begin{gathered}
\widehat{S}(\omega)=\left\{y \in Y: y=\sum_{i}^{n} \lambda_{i} u_{i}(\omega), \sum_{i}^{m} \lambda_{i}=1, \lambda_{i} \geq 0, i=1, \ldots, n\right\}, \\
\left\langle\alpha(\omega), u_{j}(\omega)\right\rangle=0, \quad j=m+1, \ldots, n, \\
\left\langle\alpha(\omega), u_{i}(\omega)\right\rangle=\psi(\omega), \quad i=1, \ldots, m,
\end{gathered}
$$

for every $\omega \in U$. In particular, $\psi$ is of class $C^{r}$ on $U$.

Proof. The technique used in the proof of Proposition 3.1 goes through without change.

Duality relations between the primal and dual problems can be seen in the next proposition.

Proposition 3.3. For every open set $U \subseteq \Omega$, there exists an open subset $U \subseteq U$ such that exactly one of the following holds

(i) $M(\omega)=\varnothing$ and $\widehat{M}(\omega)=\varnothing$ for all $\omega \in U$;

(ii) $M(\omega)=\varnothing$ for all $\omega \in U$, and there can be found a function $u \in C^{r}(\Omega, Y)$ satisfying (5);

(iii) $\widehat{M}(\omega)=\varnothing$ for all $\omega \in U$, and there can be found a function $v \in C^{r}(\Omega, X)$ satisfying $(1)$;

(iv) $M(\omega) \neq \varnothing, \widehat{M}(\omega) \neq \varnothing$ for all $\omega \in U$, and there can be found l functions $v, \ldots, v_{l} \in C^{r}(U, X) ; n$ functions $u, \ldots, u_{n} \in C^{r}(U, Y)$ and two integers $m, k$ with $0<m \leq n, 0<k \leq l$ such that (2)-(4) and (6)-(8) hold. In this event $\phi(\omega)=\psi(\omega)$ for every $\omega \in U$.

Proof. Applying Proposition 2.1 to $M$ and $\widehat{M}$ we can find an open subset $U \subseteq U$ such that either (i) holds, or at least one of these maps has nonempty values on $U$.

If $M(\omega)=\varnothing$ while $\widehat{M}(\omega) \neq \varnothing$ for all $\omega \in U$, by duality, $\psi(\omega)=\infty$ for all $\omega \in U$. Since $\widehat{M}$ is also determined by a parametric system of linear inequalities and equations, by Proposition 2.1, we may assume that it is represented by points and directions $u, \ldots, u_{n} \in C^{r}(U, Y)$. Now, the second conclusion follows from Proposition 3.2. The third one is proven dually. For the last conclusion, observe 
that if both $M(\omega)$ and $\widehat{M}(\omega)$ are nonempty, by duality, the two optimal values must be finite and equal. The result is then derived from Propositions 3.1 and 3.2.

It should be noted that since the optimal solution set of the dual problem is exactly the Kuhn-Tucker multiplier set of the primal problem ([14]), it follows from (iv) of Proposition 3.3 that if the primal problem $P(\omega)$ possesses optimal solutions for all $\omega \in U$, its Kuhn-Tucker multiplier set admits representation of class $C^{r}$ on an open dense subset of $U$.

As a consequence of Proposition 3.3 we also have the following parametric version of Farkas' theorem (see also [8] for the measurable case). For the sake of simple presentation, suppose that $M(\omega)$ is defined by a system of inequalities only, which corresponds to the case $q=0$. Given a vector $u:=\left(u, \ldots, u_{n}\right) \in R^{n}$, by writing $u \geq 0$ we mean $u_{i} \geq 0$ for all $i=1, \ldots, n$.

Corollary 3.2. Let $a \in C^{r}\left(\Omega, X^{\prime}\right), \alpha \in C^{r}(\Omega, R)$ and assume that $M(\omega) \neq \varnothing$ and $\langle a(\omega), x\rangle+\alpha(\omega) \leq 0$ for every $x \in M(\omega)$ and for every $\omega$ from an open set $U \subseteq \Omega$. Then there exists an open subset $U \subseteq U$ and a function $u \in C^{r}(U, Y)$ such that

$$
u(\omega) \geq 0, \quad\langle u(\omega), \alpha(\omega)\rangle \leq-\alpha(\omega) \quad \text { and } \quad u(\omega) A(\omega)=a(\omega),
$$

for all $\omega \in U$.

Proof. Let us consider the linear problem $P(\omega)$ with the objective function $c(\omega):=$ $-a(\omega)$. By the hypothesis of the corollary, the feasible set of this problem is nonempty and the objective function is bounded from below on it. Hence, the optimal value $\varphi(\omega)$ is finite and minorized by $\alpha(\omega)$. By Proposition 3.3, there exists an open subset $U \subseteq U$ such that the dual $D(\omega)$ possesses optimal solutions represented in the form of (6). Pick any $u_{j}$ among $u, \ldots, u_{n} \in C^{r}(U, Y)$ and set $u:=-u_{j}$. Since $u_{j}(\omega)$ is a feasible solution of the dual problem, $u(\omega) \geq 0$, and $u(\omega) A(\omega)=a(\omega)$. Moreover, by virtue of Proposition 3.3(iv), the optimal values of the primal and dual problems are equal, hence

$$
\langle u(\omega), \alpha(\omega)\rangle=-\left\langle u_{j}(\omega), \alpha(\omega)\right\rangle=-\varphi(\omega) \leq-\alpha(\omega) .
$$

This is true for all $\omega \in U$ and the proof is complete.

\section{Parametric concave problem}

In this section let us study the following parametric concave optimization problem:

$$
\begin{aligned}
\inf & f(\omega, x), \\
\text { subject to } & x \in M(\omega)
\end{aligned}
$$

where $f \in C^{r}(\Omega \times X, R)$ is concave in the second variable whenever the first one is fixed, $M$ is as in Section 2. The marginal function $\varphi$ and the solution map are defined in the same way as in the previous section. We recall that a real function $g$ on $X$ is said to be concave (respectively quasiconcave) if for any $x, y \in X$, for any number $\lambda \in(0,1)$ one has

$$
g(\lambda x+(1-\lambda) y) \geq \lambda g(x)+(1-\lambda) g(y)
$$

(respectively, $g(\lambda x+(1-\lambda) y) \geq \min \{g(x), g(y)\})$.

One of the most important features of a concave function is that it attains its minimum on a closed convex set at boundary points if the minimum exists at 
all. This fact is still true for quasiconcave functions provided the constraint set is bounded (we refer the reader to [6] for concave optimization problems and related topics). We shall adopt the convention $\varphi(\omega)=\infty$ if $M(\omega)=\varnothing$.

Proposition 4.1. Suppose that $f \in C^{r}(\Omega \times X, R)$ is concave in the second variable for every $\omega$ in an open set $U \subseteq \Omega$. Then there exists an open subset $U$ of $U$ and a function $v \in C^{r}(U, X)$ such that exactly one of the following holds:

(i) $\varphi(\omega)=\infty$ for all $\omega \in U$;

(ii) $\varphi(\omega)=-\infty$ for all $\omega \in U$;

(iii) one can find a function $v \in C^{r}(U, X)$ such that $v(\omega)$ is a vertex of $M(\omega)$ and $\varphi(\omega)=f(\omega, v(\omega))$, for every $\omega \in U$. In particular, the marginal function is of class $C^{r}$ on $U$.

Proof. Without loss of generality we may assume that $M$ is nonvoid-valued and represented as in Proposition 2.1 (otherwise the marginal function takes the value $\infty$ on $U)$. Suppose that at some $\omega \in U, \varphi(\omega)=-\infty$. Since $f(\omega, \cdot)$ is concave, there exists $v_{i_{0}}$ for some $i \in\{1, \ldots, k\}$ and $v_{j_{0}}$ for some $j \in\{k+1, \ldots, m\}$ such that

$$
\lim _{t \rightarrow \infty} f\left(\omega, v_{i_{0}}(\omega)+t v_{j_{0}}(\omega)\right)=-\infty .
$$

We state that there exists a neighborhood $U$ of $\omega$ in $U$ such that

$$
\lim _{t \rightarrow \infty} f\left(\omega, v_{i_{0}}(\omega)+t v_{j_{0}}(\omega)\right)=-\infty,
$$

for all $\omega \in U$, which implies that $\varphi(\omega)=-\infty$ on $U$. In fact, if not, there can be found a sequence $\left\{\omega_{n}: n=1,2, \ldots\right\}$ in $U$ converging to $\omega$, such that

$$
\lim _{t \rightarrow \infty} f\left(\omega_{n}, v_{i_{0}}\left(\omega_{n}\right)+t v_{j_{0}}\left(\omega_{n}\right)\right)>-\infty,
$$

for every $n=1,2, \ldots$ Again, by the concavity of $f$ the latter inequality shows that $f\left(\omega_{n}, v_{i_{0}}\left(\omega_{n}\right)\right) \leq f\left(\omega_{n}, v_{i_{0}}\left(\omega_{n}\right)+t v_{j_{0}}\left(\omega_{n}\right)\right)$ for all $t \geq 0$. Fixing $t$ and passing to the limit when $n$ tends to $\infty$, we have $f\left(\omega, v_{i_{0}}(\omega)\right) \leq f\left(\omega, v_{i_{0}}(\omega)+t v_{j_{0}}(\omega)\right)$, which contradicts (9). Hence, in fact such $U$ exists.

For the case where the optimal value is finite on $U$, let us denote by $\bar{J}(\omega) \subseteq$ $\{1, \ldots, k\}$ the index set of representing points of $M(\omega)$ at which the objective function attains its minimum. It is evident that $\bar{J}(\omega)$ is nonempty on $U$. By the continuity of $f$, for any fixed $\omega \in U$, there exists a neighborhood $U$ of $\omega$ in $U$ such that $\bar{J}(\omega) \subseteq \bar{J}(\omega)$ for all $\omega \in U$. Hence, if we choose $\omega$ a point with

$$
|\bar{J}(\omega)|=\min \{|\bar{J}(\omega)|: \omega \in U\},
$$

then $\bar{J}(\omega)=\bar{J}(\omega)$ for all $\omega \in U$. Fixing any $i \in \bar{J}(\omega)$ we have $\varphi(\omega)=$ $f\left(\omega, v_{i_{0}}(\omega)\right)$, for every $\omega \in U$ and the proof is complete.

For quasiconcave problems we have the following result. 
every fixed $\omega \in U$, the function $f(\omega, \cdot)$ attains its minimum at some vertices. The set $\bar{J}(\omega)$ defined in the proof of Proposition 4.1 is nonempty for every $\omega \in U$, and the technique used there goes through without change.

\section{PARAMETRIC POLYHEDRAL PROBLEM}

The fact that the optimal values in the problems attacked in the two preceding sections can be attained at some vertices of the constraint domains, makes it possible to characterize solution sets by using the representation established in Proposition 2.1. In this section we consider another type of problems which also can lead to this situation. Namely, we shall study problems with polyhedral convex objective and linear constraints. Recall that a convex function is said to be polyhedral if its epigraph is polyhedral, i.e. is the intersection of finitely many closed half-spaces in $X \times R$ which are either vertical or the epigraphs of affine functions (see [14]). Analytically, a polyhedral convex function $f$ on $X$ can be given as

$$
f(x)=\max \left\{\left\langle b_{i}, x\right\rangle+\beta_{i}: i=1, \ldots, s\right\}+\delta_{N}(x),
$$

where $b, \ldots, b_{s} \in X^{\prime} ; \beta, \ldots, \beta_{s} \in R$, and $\delta_{N}(x)$ is the indicator function of a polyhedral set $N \subseteq X$.

From now on we assume that $b, \ldots, b_{s}, \beta, \ldots, \beta_{s}$ are functions of the parameter $\omega \in \Omega$ and of class $C^{r}$, and that $N$ is given by a parametric system of inequalities and equations whose entries are of class $C^{r}$ too. For us $M$ is as in Section 2. Let us consider the following problem, denoted by $P(\omega)$ :

$$
\begin{aligned}
\inf & f(\omega, x), \\
\text { subject to } & x \in M(\omega) .
\end{aligned}
$$

The marginal function $\varphi$ and the solution map $S$ are defined in the same way as in the previous sections. Note that in this problem $f$ is continuous, but generally not differentiable in the second variable. Moreover, the solution set $S(\omega)$ may contain no vertices of $M(\omega)$. To overcome these difficulties, let us consider the "extended" problem, denoted by $\widehat{P}(\omega)$ :

$$
\begin{aligned}
\inf & t, \\
\text { subject to } & (x, t) \in \widehat{M}(\omega)
\end{aligned}
$$

where $\widehat{M}(\omega)$ is the intersection of the set $M(\omega) \times R$ with the epigraph of $f(\omega, \cdot)$. The marginal function of $\widehat{P}(\omega)$ is denoted by $\hat{\varphi}$ and its solution map is denoted by $\widehat{S}$.

It is evident that $\widehat{M}(\omega)$ is a polyhedral convex set and is determined by a system of inequalities and equations whose entries are of class $C^{r}$. We still keep the convention that the optimal value of a minimization problem is $+\infty$ if the constraint set is empty. The following result is standard.

Lemma 5.1. For a fixed parameter $\omega \in \Omega$, we have the following:

(i) $\hat{\varphi}(\omega)=\varphi(\omega)$;

(ii) a point $x \in X$ is an optimal solution of $P(\omega)$ if and only if $(x, f(\omega, x))$ is an optimal solution of $\widehat{P}(\omega)$.

Proof. Observe that if $M(\omega)$ is empty, then so is $\widehat{M}(\omega)$. Conversely, if $\widehat{M}(\omega)$ is empty, then either $M(\omega)$ is empty, or $f(\omega, \cdot)$ takes values $+\infty$ on it. Hence, $\hat{\varphi}(\omega)=+\infty$ if and only if $\varphi(\omega)=+\infty$. 
Now, if $\varphi(\omega)=-\infty$, one can find a sequence $\left\{x_{n}: n=1, \ldots\right\}$ in $M(\omega)$ such that $\lim _{n \rightarrow \infty} f\left(\omega, x_{n}\right)=-\infty$. This implies that $\hat{\varphi}(\omega)=-\infty$, because the sequence $\left\{\left(x_{n}, f\left(\omega, x_{n}\right)\right): n=1, \ldots\right\}$ belongs to $\widehat{M}(\omega)$. A similar argument shows that $\hat{\varphi}(\omega)=-\infty$ implies $\varphi(\omega)=-\infty$.

Furthermore, if $x$ is an optimal solution of $P(\omega)$, then $f(\omega, x) \leq f(\omega, x) \leq t$, for every $x \in \widehat{M}(\omega), t \geq f(\omega, x)$. Hence $(x, f(\omega, x))$ is an optimal solution of $\widehat{P}(\omega)$ and both of the optimal values are equal. The converse part is proven in a similar way.

Proposition 5.1. For every open set $U \subseteq \Omega$, there exists an open subset $U \subseteq U$ such that exactly one of the following holds:

(i) $\varphi(\omega)=+\infty$ for all $\omega \in U$;

(ii) there exist functions $v, v \in C^{r}(\Omega, X)$ such that

$$
\begin{gathered}
v(\omega) \in M(\omega), v(\omega) \in \operatorname{Rec} M(\omega), \\
\lim _{t \rightarrow \infty} f(\omega, x v(\omega)+t v(\omega))=-\infty
\end{gathered}
$$

for all $\omega \in U$;

(iii) there exist l functions $v, \ldots, v_{l} \in C^{r}(U, X)$, an integer $k$ with $0<k \leq l$, and some $j \in\{1, \ldots, s\}$ such that

$$
\begin{gathered}
v(\omega), \ldots, v_{k}(\omega) \in M(\omega), \quad v_{k} \quad(\omega), \ldots, v_{l}(\omega) \in \operatorname{Rec} M(\omega), \\
S(\omega)=\left\{\begin{array}{l}
l \\
\left.x \in X: x=\sum_{i}^{l} \lambda_{i} v_{i}(\omega), \sum_{i}^{k} \lambda_{i}=1, \lambda_{i} \geq 0, i=1, \ldots, l\right\}, \\
\left\langle b_{j}(\omega), v_{i}(\omega)\right\rangle+\beta_{i}(\omega)=\varphi(\omega), \quad i=1, \ldots, k,
\end{array}\right.
\end{gathered}
$$

for every $\omega \in U$. In particular, the marginal function is of class $C^{r}$ on $U$.

Proof. In view of Proposition 2.1, there exists an open subset $U$ in $U$ such that either $\widehat{M}(\omega)$ is empty for all $\omega \in U$, or it is nonvoid-valued there. The first case corresponds to the first assertion of the proposition. In the other case we apply Proposition 3.1 to Problem $\widehat{P}(\omega)$. There exists an open subset $U \subseteq U$ such that either (1) or (2)-(4) hold. We use "hat" over the corresponding functions in these formulas for Problem $\widehat{P}(\omega)$. Thus, in our case, by $(1), \hat{v}(\omega):=(v(\omega), \tau(\omega))$ is a recession direction of $\widehat{M}(\omega)$, satisfying the inequality $\tau(\omega)<0$. It is clear that $v(\omega)$ is a recession direction of $M(\omega)$. Moreover, let $\widehat{v}(\omega):=(v(\omega), \tau(\omega))$ be the first point in the representation of $\widehat{M}(\omega)$ according to Proposition 2.1. Then $v(\omega) \in M(\omega)$, and $\lim _{t \rightarrow \infty} f(\omega, v(\omega)+t v(\omega)) \leq \lim _{t \rightarrow \infty}(\tau(\omega)+t \tau(\omega))=-\infty$. This is true for all $\omega \in U$, hence (ii) follows by setting $U:=U$.

In the case where the optimal value is finite, as above, we express $\hat{v}_{i}(\omega)=$ $\left(v_{i}(\omega), \tau_{i}(\omega)\right), i=1, \ldots, l$. Here too, one sees that $v(\omega), \ldots, v_{k}(\omega) \in M(\omega)$ and $v_{k} \quad(\omega), \ldots, v_{l}(\omega) \in \operatorname{Rec} M(\omega)$. By Lemma 5.1, the solution set of $P(\omega)$ is represented by the points $v(\omega), \ldots, v_{k}(\omega)$ and the directions $v_{k}(\omega), \ldots, v_{l}(\omega)$. Furthermore, let $I(\omega)$ be the set of all the indices $i \in\{1, \ldots, s\}$ which satisfy the relation $\left\langle b_{i}(\omega), v_{j}(\omega)\right\rangle+\beta_{i}(\omega)=\hat{\varphi}(\omega)$ for $j=1, \ldots, k$. Then if we choose a point $\omega \in U$ with the property that $|I(\omega)|=\min \{|I(\omega)|: \omega \in U\}$, it follows that the sets $I(\omega)$ and $I(\omega)$ coincide for all $\omega$ in a sufficiently small neighborhood $U$ of $\omega$ in $U$. Pick any $j$ among $I(\omega)$ to obtain (10). The proof is complete. 


\section{ACKNOWLEDGEMENTS}

This paper was partly written during the author's stay at the Universitat Autonoma de Barcelona. The author would like to express his thanks to Professors I. Fradera and J.-E. Martinez-Legas for their kind invitation and hospitality.

\section{REFERENCES}

[1] J.-P. Aubin and H. Frankowska, Set-valued Analysis, Birkhäuser, Boston, MA, 1990. MR 91d:49001

[2] B. Bank, J. Guddat, D. Klatte, B. Kummer, K. Tammer, Nonlinear Parametric Optimization, Akademie-Verlag, Berlin, 1982, and Birkhäuser, Boston, MA, 1983. MR 84i:90147

[3] C. Berge, Topological Spaces, Macmillan, New York, 1963. MR 21:4401 (French original).

[4] G. B. Dantzig, J. Folkman and N. Shapiro, On the continuity of the minimum sets of a continuous function, J. Math. Anal. Appl. 17 (1967), 519-548. MR 34:7241

[5] A. V. Fiacco, Introduction to Sensitivity and Stability Analysis in Nonlinear Programming, Academic Press, New York, 1983. MR 85b:90072

[6] R. Horst and H. Tuy, Global Optimization, Springer-Verlag, Berlin, 1990. MR 92d:90002

[7] A. B. Levy and R. T. Rockafellar, Sensitivity analysis of solutions to generalized equations, Trans. Amer. Math. Soc. 345 (1994), 661-671. MR 95a:90164

[8] D. T. Luc, Random version of the theorems of the alternative, Math. Nach. 129 (1986), 149-155. MR 88d:46087

[9] D. T. Luc and P. H. Dien, Differentiable selection of optimal solutions in parametric linear programming, Proc. Amer. Math. Soc. (in press).

[10] J.-P. Penot, Preservation of persistence and stability under intersections and operations, Parts I, II, J. Optim. Theory Appl. 79 (1993), 525-550, 551-561.

[11] J.-P. Penot, Compact nets, filters and relations, J. Math. Anal. Appl. 93 (1983), 400-417. MR 84h: 49032

[12] S. M. Robinson, Stability theory for systems of inequalities. Part I: Linear systems, SIAM J. Numer. Anal. 12 (1975), 754-769. MR 53:14270

[13] S. M. Robinson, An implicit-function theorem for a class of nonsmooth functions, Math. Oper. Res. 16 (1991), 292-304. MR 92g:58013

[14] R. T. Rockafellar, Convex Analysis, Princeton Univ Press, Princeton, NJ, 1970. MR 43:445

Université D'Avignon, 33 rue Louis Pasteur, Avignon, France

Current address: Institute of Mathematics, P. O. Box 631, Hanoi, Vietnam 